\title{
Experimental Approach on Concentration Measurements of NO in Hydrogen Combustion Based on Heterodyne Laser Absorption Spectroscopy Using Quantum Cascade Lasers
}

\author{
Jan Martinez Schramm ${ }^{(凶)}$ and Diana Luís \\ Department Spacecraft, German Aerospace Center, Institute of Aerodynamics and \\ Flow Technology, Bunsenstraße 10, 37073 Göttingen, Germany \\ \{Jan.Martinez, Diana.Luis\}@dlr.de
}

\begin{abstract}
Experimental determination of $\mathrm{NO}$ and $\mathrm{H}_{2} \mathrm{O}$ production during hydrogen combustion applying laser absorption spectroscopy will be performed in the High Enthalpy Shock Tunnel Göttingen of the German Aerospace Center. The combustion process forming these species within the propulsion unit of a small scale wind tunnel model is to be studied. A novel experimental approach to obtain absorption spectra of both species in the infrared region within $\mu$-second range is reported. This technique is based on the use of frequency combs generated with quantum cascade lasers. This method enables multi-species measurements of absorption spectra from which concentrations can be inferred. The generated frequency combs are within the $1760 \pm 30 \mathrm{~cm}^{-1}$ wavenumber region which features strong $\mathrm{NO}$ and $\mathrm{H}_{2} \mathrm{O}$ spectral lines allowing characterization of these species. The technique, the application in short duration ground testing and its calibration procedure will be reported.
\end{abstract}

Keywords: HEG $\cdot$ H2020 $\cdot$ STRATOFLY $\cdot$ Laser absorption spectroscopy $\cdot$ Hydrogen combustion $\cdot$ SCRAMJET $\cdot$ Dual comb frequency - Quantum cascade lasers

\section{Introduction}

A worldwide growing willingness to dramatically reduce the environmental impact of civil aviation within the next decades is noticeable. At the same time the need to improve the current civil transport aircraft performance is urging for the development of new high speed vehicles. The H2020 STRATOFLY (Stratospheric Flying Opportunities for High Speed Propulsion Concepts) project funded by the European Commission under the European Union's Horizon 2020 research and innovation programme was set up to study the feasibility of high speed passenger stratospheric flight. One main goal is to drastically decrease the transfer time of long range civil flights which implies performing an evaluation of

(C) The Author(s), under exclusive license to Springer Nature Switzerland AG 2021

A. Dillmann et al. (Eds.): STAB/DGLR Symposium 2020, NNFM 151, pp. 110-120, 2021.

https://doi.org/10.1007/978-3-030-79561-0_11 
the economic sustainability of the future operation of hypersonic vehicles. The use of hydrogen combustion based scramjets as propulsion units is one possibility to drive hypersonic passenger transport. It is scientifically well established that cruise $N O$ emissions cause a significant part of the current total climate impact of aviation [1]. The experimental determination of $\mathrm{NO}$ and $\mathrm{H}_{2} \mathrm{O}$ production in hydrogen combustion is part of the contribution of the German Aerospace Center (DLR) to the STRATOFLY project. The measurements are performed in the High Enthalpy Shock Tunnel Göttingen (HEG), one of the major European hypersonic test facilities. The test time for experiments in HEG is typically in the $m s$ range, which requires diagnostic techniques with high acquisition rates. The combustion process within the propulsion unit of a small scale flight experiment wind tunnel model will be studied employing heterodyne dual frequency comb based absorption spectroscopy with quantum cascade lasers (QCLs) in the infrared region within $\mu$-second range. The paper reports on this novel approach, describes the technical and experimental prerequisites, and demonstrates the ability of this technique in the context of the measurement task.

\section{Dual Comb Laser Absorption Spectroscopy}

The process which led to the selection of the laser absorption spectroscopy based on the heterodyne processing of dual comb laser beating signals is explained in this section. The theoretical absorption spectra expected based on numerical flow simulations are shortly introduced. From here, the heterodyne technique allowing the detection of IR wavelength absorption spectra in the $\mu s$ range and the QCL (quantum cascade laser) hardware enabling this are discussed.

Figure 1 (left) presents a broad $\mathrm{NO}$ and $\mathrm{H}_{2} \mathrm{O}$ absorption spectra for the hydrogen combustion flow case to be studied ( $p=4.14$ bar, $T=2320 \mathrm{~K}$ ). More details about the flow case are given in Sect.3.2. The strongest NO absorption lines expected in the planned combustion experiment occur in a $1500 \mathrm{~cm}^{-1}-2000 \mathrm{~cm}^{-1}$ region. The QCLs chosen for this work have spectral coverage of $1730 \mathrm{~cm}^{-1}-1790 \mathrm{~cm}^{-1}$. Figure 1 (right) shows a feature of the expected absorption spectra in the hydrogen combustion flow for $\mathrm{NO}$ and $\mathrm{H}_{2} \mathrm{O}$ in the QCL emission region. The simulated spectra are overlaid with symbols to indicate the spectral resolution of the spectrometer. It is concluded that the absorption of $\mathrm{H}_{2} \mathrm{O}$ and $\mathrm{NO}$ can be separated and the reconstruction of the state of both species can be performed with one measurement. The challenge is to obtain IR-absorption spectra at high repetition rates. The constant flow state of the planned experiment will be in the $m s$ range and thus the acquisition rates of the spectrometer have to be in the $\mu s$ range. The optical frequency comb technique allows for that.

A significant challenge in the field of optical frequency measurement is to obtain values with high accuracy, as light frequencies oscillate $10^{5}$ times faster than state of the art digital electronics can resolve [3]. Up to the millennium, methods to access an optical frequency relied on wavelength determination and on the knowledge of the speed of light [3], which lead to poor precision. Around 

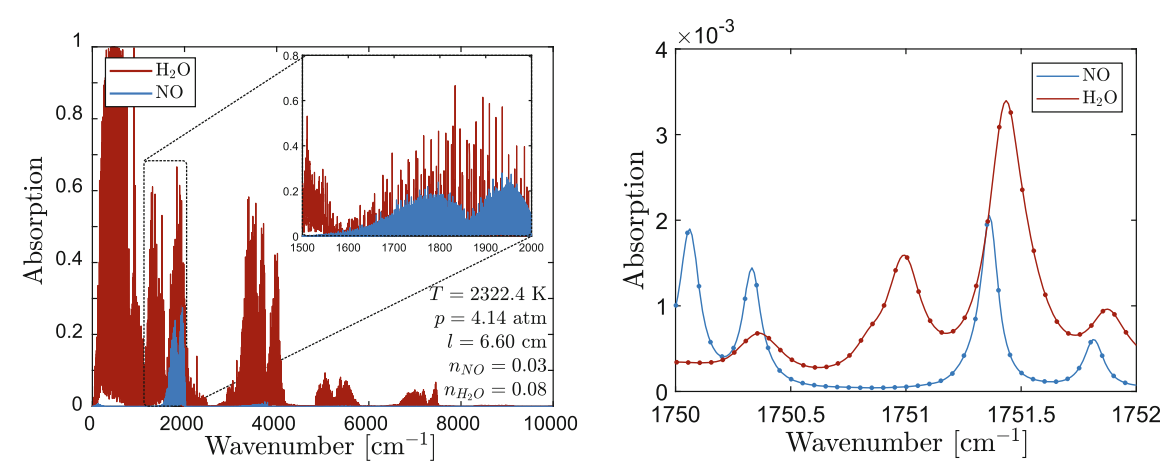

Fig. 1. Simulated $\mathrm{NO}$ and $\mathrm{H}_{2} \mathrm{O}$ absorption spectra for a representative gas state during hydrogen combustion ( $p=4.14$ bar, $T=2320 \mathrm{~K}$ ) obtained using HITRAN/HAPI [2]. Left: Broad spectra. Right: Feature of the expected spectra with points corresponding to the wavenumber resolution of the spectrometer.

2000, John L. Hall and Theodor W. Hänsch developed the frequency comb technique in which laser light with a series of equidistant frequencies is used to measure frequencies with great precision. For this accomplishment, the two scientists were awarded the Nobel Prize of Physics in 2005 for "their contributions to the development of laser-based precision spectroscopy, including the optical frequency comb technique" [4].

The application of this technique allows the determination of optical difference frequencies with good accuracy. These difference frequencies in the infrared light region can be used because they fall within the bandwidth limit of precision frequency counters $(<10 \mathrm{GHz})[3]$. Consider as a simplified example, two optical frequency comb carriers with slightly different repetition frequencies $f_{r e p, 1}$ and $f_{r e p, 2}$, shown by the blue and red lines in Fig. 2 on the left. The combination of these combs can be treated as an optical carrier with an amplitude modulation at the frequency difference, $\Delta f_{r e p}=f_{r e p, 2}-f_{r e p, 1}$. When these laser signals are aligned on a photo detector, the detector produces a voltage proportional to the amplitude modulation, referred as heterodyne beating frequency. This heterodyne beating signal occurs in a much smaller frequency region allowing to capture its frequency contents by analyzing the photo detector signals (green line in Fig. 2 left). A single optical frequency comb consists of a very regular pattern of sharp spectral lines in the frequency domain. The comb frequency lines [5] at frequencies $f_{n}$, given by

$$
f_{n}=n f_{r e p}+f_{o}
$$

are spaced by the repetition frequency $f_{\text {rep }}$ and shifted by the offset frequency $f_{o}$, satisfying $f_{o}<f_{r e p}$. Both $f_{r e p}$ and $f_{o}$ are in the radio frequency (RF) range, while the mode number $n$ is large enough to scale $f_{n}$ to optical frequencies [6].

Optical frequency combs are conventionally generated at the output of a mode-locked laser that emit short pulses. A combination of successive 


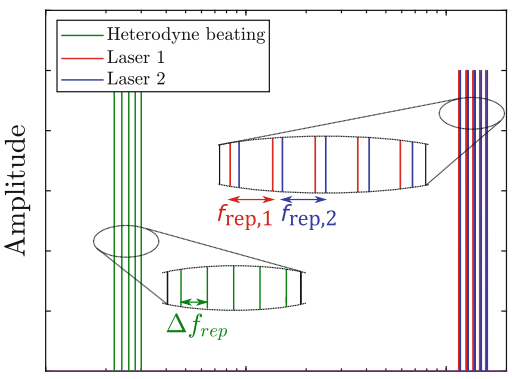

Frequency $[\mathrm{Hz}]$

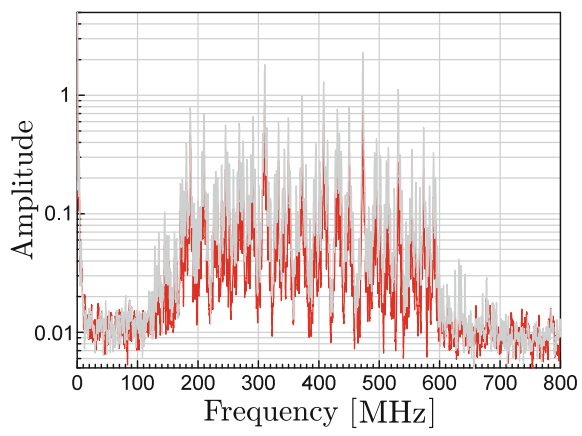

Fig. 2. Left: Two frequency combs with slightly different repetition frequencies (blue and red lines) and the resulting heterodyne beating frequency (green lines) observed when both frequency combs are optically aligned on the same detector. Right: Realistic beating signals from the IR-lasers used. The signals show the beating of two aligned beams carrying an absorption information (in red) and two beams propagating through a $N_{2}$ purged path (in grey). (Color figure online)

longitudinal laser modes gives rise to pulses every $\tau_{\text {rep }}$ if they have the same phase. The fixed phase relationship between the optical modes is needed for the formation of the optical comb spectrum described in Eq.1. This relationship is obtained by having a single pulse propagating in the laser cavity. The round trip time $\tau_{r e p}$ is the inverse to the repetition frequency $f_{r e p}$ of the emitted pulse train. After each round-trip, the temporal pulse is exactly restored which results in a stable pulse envelope at the laser output.

The generation of optical frequency combs with QCLs lies on the intrinsic physical properties of these laser sources. QCLs are semiconductors injection lasers emitting throughout the mid-infrared $\left(416-3333 \mathrm{~cm}^{-1}\right)$ and $\mathrm{THz}(40-200$ $\mathrm{cm}^{-1}$ ) regions of the electromagnetic spectrum. A state-of-art infrared (IR) QCL laser source installed in a spectrometer (IRis-F1, IRsweep AG, Switzerland) is chosen to conduct the absorption measurements, to ultimately infer concentrations of $\mathrm{NO}$ and $\mathrm{H}_{2} \mathrm{O}$ in a supersonic hydrogen combustion flow case. The IRis-F1 spectrometer uses a four wave mixing of phase-locked QCL to fabricate stable and repeatable IR frequency combs [7]. The comb is formed from an approximately 6-mm-long indium gallium arsenide/indium aluminum arsenide (InGaAs/InAlAs) QCL cavity and has approximately 200 comb lines with $7.5 \mathrm{GHz}\left(0.25 \mathrm{~cm}^{-1}\right)$ comb-line spacing [8]. A heterodyne beating signal obtained from this device is shown in Fig. 2 on the right. The position of the frequency comb shown in Fig. 2 on the right with respect to the base frequency depends mainly on the length of the laser cavity. The length of the cavity depends on its temperature and the temperature can be controlled by the current applied. Changing the current can be used to tune the measurement to a specific absorption line. The line spacing within the comb is only weakly dependent of the varying length of the cavity. 


\section{Experimental Apparatus}

\subsection{Spectrometer}

The IRis-F1 spectrometer has two QCLs installed, with a spectral coverage of $30 \mathrm{~cm}^{-1}$ centered at $1760 \mathrm{~cm}^{-1}$. Details on the spectrometer can be found in Ref. [9]. The beam of each QCLs is attenuated with $25 \%$ transmission (0.6 optical density) neutral density (ND) filters (Thorlabs ND06A) and combined through a 50:50 $\mathrm{CaF}_{2}$ beam splitter (BS) inside the laser module as shown in Fig. 3. The beam splitter divides the light into a sample beam path which reaches the sample detector (D1), and a reference beam path which is aligned onto the reference detector (D2). The detectors are a high-bandwidth $(1 \mathrm{GHz})$ AC-coupled $\mathrm{HgCdTe}$ (Vigo PV-4TE-10.6), and are reached by the light beams through a parabolic mirror (Thorlabs MPD019-M01). Each of the detectors yield a heterodyne beating signal (grey and red signals in Fig. 2 on the right). When the sample beam passes through the combustion chamber of the model, specific frequencies of the laser are absorbed leading to a decrease of the intensity of the light beam and, consequently, the baseline heterodyne signal. To align the sample beam out and back inside the spectrometer through the combustion chamber, additional optics are used. The optical setup coupling the sample beam out of the spectrometer consists of a polarizer (Thorlabs PS810) combined with a lens which focuses the light onto an optical fiber with an FC connector at Brewster angle (IRflex IRF-Se-12). The Brewster angle minimizes back reflections and maximizes the power being coupled into the fiber. The other end of the fiber transporting the light to the model has a SMA connector inserted on a fiber coupler (Thorlabs RC02SMA-P01). A second coupler receives the beam after it passed the combustion chamber and feeds it to a second fiber, which is then coupled again on a third coupler and aligned with the parabolic mirror inside of the spectrometer. An external electronic box (current driver) from IRsweep is used to control the current driving the lasers.
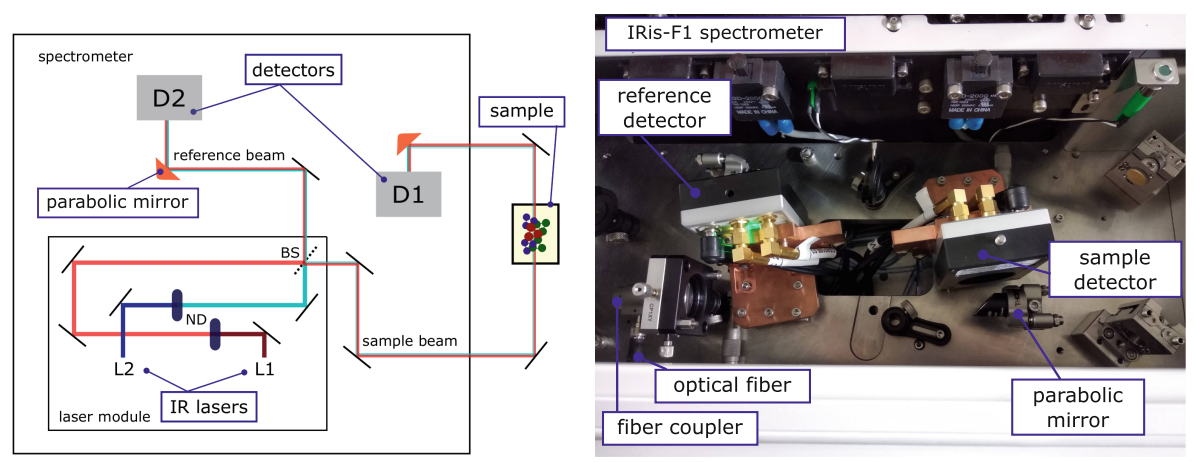

Fig. 3. Left: Simple schematic of the beam paths. Right: Actual setup inside of the spectrometer for detection of the laser beams. 
The spectrometer allows three different types of processing modes: static, time resolved and sweeping. In static mode, a single spectrum is obtained from averaging multiple number of measurements; in time resolved mode, the multiple measurements are taken and saved, yielding a spectrum for each time instant. The static and time resolved modes leave the lasers current constant throughout the acquisition allowing time resolutions at $4 \mu \mathrm{s}[9]$ and a spectral point spacing of $0.25 \mathrm{~cm}^{-1}$. In sweeping mode the current of the lasers is periodically modulated in a saw-tooth wave (Fig. 4) at a rate of $5 \mathrm{~mA} / \mathrm{V}$, by a wave generator. Each ramp is set to increase from $0 \mathrm{~V}$ to approximately $6 \mathrm{~V}$ and takes about $1 \mathrm{~ms}$ to sweep through. The exact maximum voltage value is chosen by fine tuning so that the heterodyne beating signal is stable and well defined. The ramps are recorded and thus, the frequency changes can be precisely coordinated in the post processing. This method decreases the time resolution of the measurements, but increases the spectral resolution by a factor of almost 250 . This is a theoretical value when sampling with $4 \mu$ s during $1 \mathrm{~ms}$ that is checked experimentally as well.

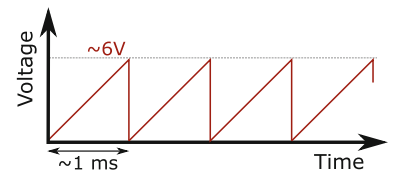

Fig. 4. Saw-tooth wave

To perform calibration in the tunnel, the sample beam is aligned through the model, the spectrometer is purged with $N_{2}$, the test section is in vacuum, and the lasers conditions and instrument parameters are the same as during the experimental test. Ahead of the measurements, transfer functions are taken with the lasers light blocked. These functions are used as background and help eliminating the electronic noise of the system when there is no light on the detectors. During calibration, compressed room air is injected by the hydrogen supply system into the combustion chamber. The measurement is done in time resolved processing mode and triggered by the hydrogen injection trigger line. The pre-trigger time is used as a background measurement as the test section and, consequently, the combustion chamber, are in vacuum. The comparison between the spectra before and after the trigger allows observing the $\mathrm{H}_{2} \mathrm{O}$ content on the compressed air. Fitting the $\mathrm{H}_{2} \mathrm{O}$ spectrum obtained experimentally with the theoretical water spectrum generated with HAPI [2] allows calibration of the wavenumber axis (see Sect. 3.4).

\subsection{HEG and SSFE Wind Tunnel Model}

Experiments will be conducted in the High Enthalpy Shock Tunnel (HEG) of the German Aerospace Center, DLR. HEG is designed on the working principle of a free piston driven shock tube (Stalker tube [10]) and is one of the major European hypersonic test facilities of this kind. Additional information about the working principle of the facility, its capabilities and former use in combustion research can be found in Ref. [11,12]. The operating condition that will be used for the experimental campaign is condition H3.3R3.7 (former condition XIII). Table 1 lists the nominal reservoir conditions for the nozzle, the Pitot pressure $p_{t 2}$ and stagnation point heat flux $q_{t 2}$, as well the complete set of free stream parameters. The subscript 0 refers to the reservoir conditions and $t 2$ to the total conditions behind the normal shock. The other variables $p$ yield pressure, $T$ temperature, $h$ specific enthalpy, $u$ velocity, Ma Mach number, $R e$ 
Reynolds number, $\rho$ density, and $m_{\mathrm{O}_{2}}$ mass flow rate of oxygen. The total available test time is approximately $5 \mathrm{~ms}$. Based on the nozzle reservoir conditions, the free stream is determined by numerical computation of the nozzle flow [12]. The nozzle reservoir gas temperature is quite low, when the total simulation range of HEG is considered. Typical problems in these facilities related to the development of sooth in the freestream leading to dimming of light traversing through the gas are not expected for the planned experiments. A final check has still to be done when the experiments are performed. Another typical and challenging problem for optical measurement techniques in shock tunnels is the free gas radiation (self-illumination of the flow). Pre-tests did not show any emission in the wavenumber region worth considering for the laser absorption technique.

Table 1. HEG operating conditions

\begin{tabular}{c|c|c|c|c|c|c}
$p_{0}[\mathrm{MPa}]$ & $T_{0}[\mathrm{~K}]$ & $h_{0}[\mathrm{MJ} / \mathrm{kg}]$ & \multicolumn{2}{c|}{$p_{t 2}[\mathrm{kPa}]$} & $q_{t 2}\left[\mathrm{MW} / \mathrm{m}^{2}\right]$ \\
\hline 170 & 2740 & \multicolumn{2}{|c}{3.3} & 146.53 & 3.71 \\
$p[\mathrm{~Pa}]$ & $T[\mathrm{~K}]$ & $\rho\left[\mathrm{g} / \mathrm{m}^{3}\right]$ & $u[\mathrm{~m} / \mathrm{s}]$ & $m_{O_{2}}\left[\mathrm{~g} / \mathrm{m}^{3}\right]$ & $\mathrm{Ma}[-]$ & $\operatorname{Re}[1 / \mathrm{m}]$ \\
\hline 1990 & 266 & 25.9 & 2398 & 6.34 & 7.4 & $3.7 \cdot 10^{-6}$
\end{tabular}

The supersonic hydrogen combustion flow to be studied will be generated in the propulsion unit of the SFFE wind tunnel model. SSFE stands for small scale flight experiment. This configuration is based on the full scale LAPCAT (Long-Term Advanced Propulsion Concepts and Technologies) II Mach 8 MR 2 vehicle proposed by ESA-ESTEC as described in Ref. [13]. The MR 2 vehicle is characterized by the combination of an aerodynamic efficient wave rider configuration and the dorsal integration of a high performance propulsion unit whose cruise and aero-propulsive performance was numerically assessed [14]. The SSFE configuration was derived from the MR2 as reported in Ref. [15]. The combustor layout is shown in Fig. 5. Two semi strut and one full strut injector expand the hydrogen into the combustor. The generation of $N O$ in the combustion process is visualised by iso surfaces from the numerical rebuilding of the combustor flow. Additionally the lines of sight for the laser absorption spectroscopy measurements are shown. Further details about the model design are given in Ref. [16]. 


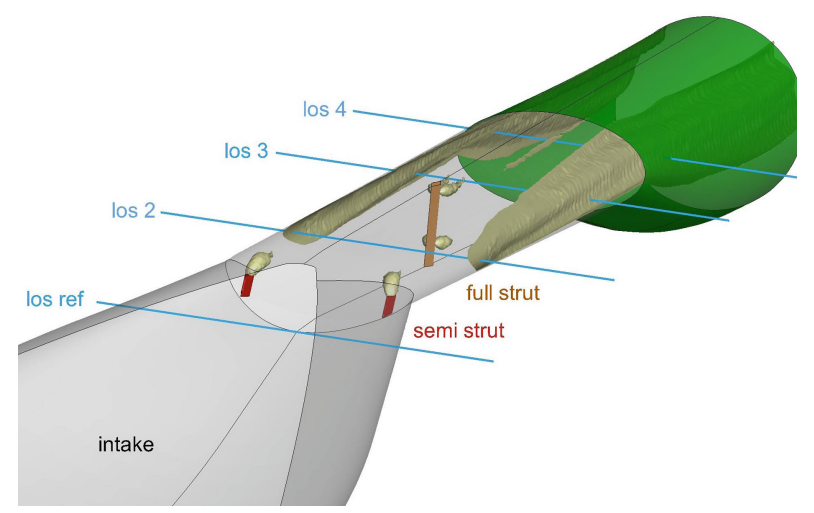

Fig. 5. CFD simulation showing iso surface for $N O$ (mass fraction of 0.1).

To couple the laser into the model and to allow measurements along the lines of sight, eight optical ports were manufactured into the model. Each of these ports contains a Zinc-Selenide (ZnSe) window. The optical parts coupling the infrared light into the combustion chamber are mounted on and supported by an additional optical rail fixed outside to the model as pictured in Fig. 6 .

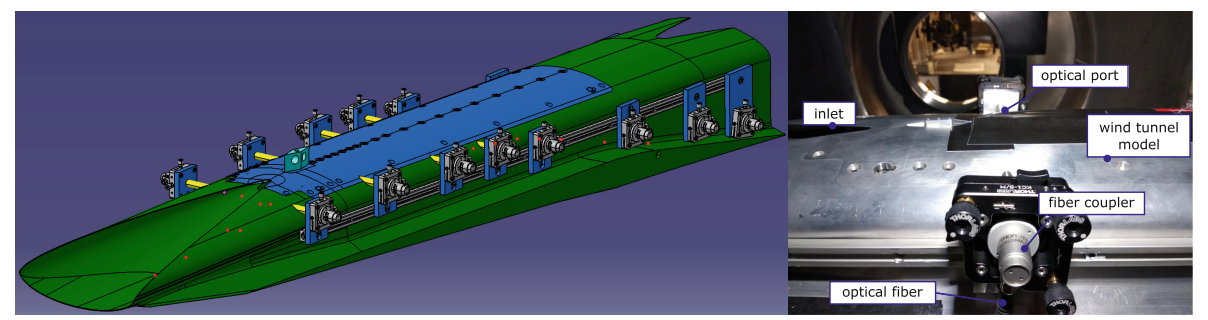

Fig. 6. Left: Schematic of the SSFE model with the optical couplers installed. Right: Photograph of an optical coupler installed on the model.

\subsection{Flow State of the SSFE Propulsion Unit}

The numerical flow properties along the los 2 coordinate $Y$ are given in Fig. 7 on the left. The distribution of temperature $T$, pressure $p$ and the mass fractions for $\mathrm{H}_{2} \mathrm{O}$ and $\mathrm{NO}$ are shown. Two different procedures have been pursuit to obtain the resulting $\mathrm{NO}$ and $\mathrm{H}_{2} \mathrm{O}$ absorption spectra using HITRAN/HAPI [2]. The first assumes that the gas properties at the symmetry axis are constant throughout the line of sight; the latter uses a more sophisticated determination procedure. Here, individual spectra are obtained using the varying gas properties along the line of sight and the distances between the individual points are considered as integration length. All spectra are then integrated to give a resulting 
spectrum. The comparison between both procedures is shown in Fig. 7 on the right. The solid lines denote the results for the first procedure and the dotted lines the results for the latter. There is a notable difference between both procedures and thus the integration through the complete flow field is necessary, due to the large variation of the gas properties perpendicularly to the flow direction.
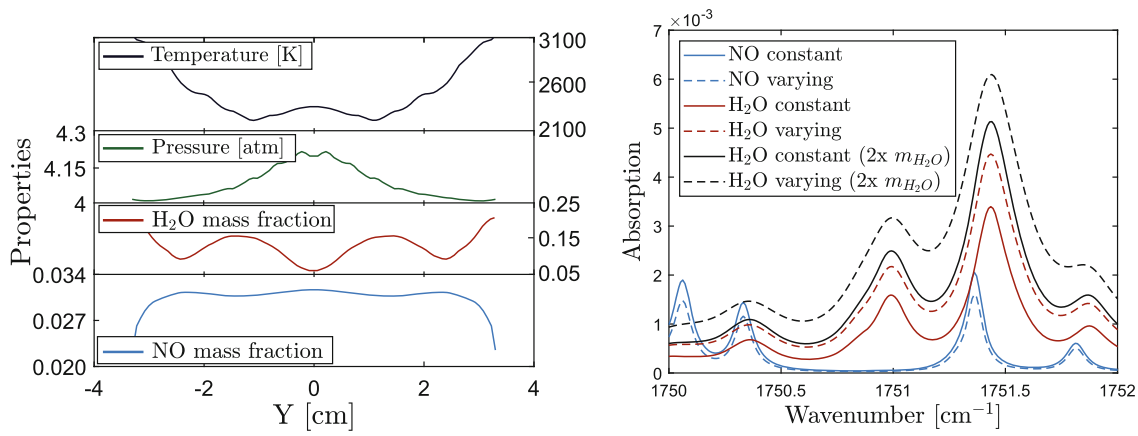

Fig. 7. Left: Flow properties (temperature, pressure, and $\mathrm{NO}$ and $\mathrm{H}_{2} \mathrm{O}$ mass fractions) along the los 2 extracted from the numerical solution. Right: Simulated $\mathrm{NO}$ and $\mathrm{H}_{2} \mathrm{O}$ absorption spectra using constant properties from the axis symmetry (solid lines) and properties varying (dashed lines) both along the los 2 .

\subsection{Wavenumber Calibration}

Some preliminary calibration measurements are conducted under lab conditions to test the technique, prior to the experimental campaign at HEG. Measurements are taken with atmospheric air introduced into the sample path after obtaining the background measurements. Figure 8 presents some calibrated experimental points within a water feature (black dots) using sweeping mode. The calibrated wavenumber is obtained by moving the data along the wavenumber axis to fit the theoretical results obtained with HAPI [2]. In Fig. 8 (left), the spectral resolution in time resolved mode is highlighted and the number of points measured in between a $0.25 \mathrm{~cm}^{-1}$ interval is visible. As previously mentioned, the spectral resolution of the measurements increases when using the sweeping mode.

A parametric study is conducted to analyse the variation of the theoretical spectrum with pressure (Fig. 8 left), temperature (Fig. 8 center), and $\mathrm{H}_{2} \mathrm{O}$ concentration (Fig. 8 right). It is observed that small changes in temperature do not influence significantly the transmission spectrum. The most significant parameter is the concentration. To obtain an impression of the flow case investigated, an increase by double of the concentration of $\mathrm{H}_{2} \mathrm{O}$ is shown in Fig. 7 on the right (black lines). A change in roughly $25 \%$ is expected in the absorption signal. Additional and optimized measurements that will access other absorption lines of water and subsequently $N O$ will be performed as the establishment of a well defined calibration method is one of the most important prerequisites before the experimental campaign at HEG. 

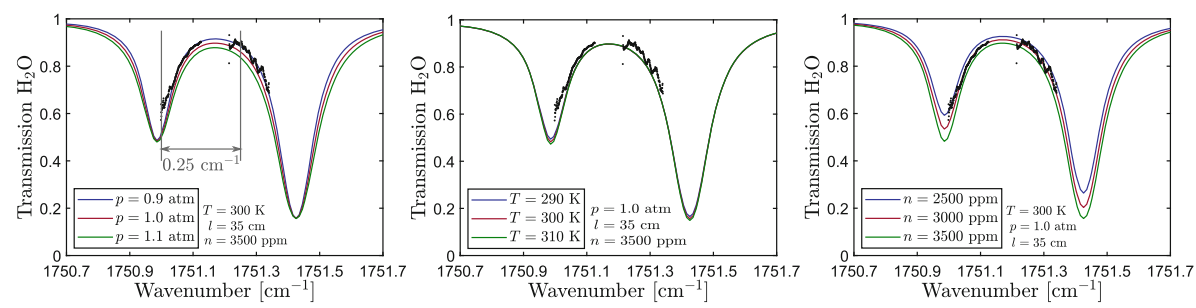

Fig. 8. Feature of experimental data using sweeping mode when measuring water (black dots) and fit with numerical results from HAPI [2]. Left: Variation of the theoretical $\mathrm{H}_{2} \mathrm{O}$ transmission spectrum with pressure. Detail on the spectral resolution with time resolved mode. Center: Variation of the spectrum with temperature. Right: Variation of the spectrum with $\mathrm{H}_{2} \mathrm{O}$ mole fraction.

\section{Conclusions}

Experimental determination of $\mathrm{NO}$ and $\mathrm{H}_{2} \mathrm{O}$ production during hydrogen combustion is to be performed by employing laser absorption spectroscopy. This novel experimental approach to obtain absorption spectra of both species in the infrared region within $\mu$-second range is reported. This technique is based on the use of frequency combs generated with quantum cascade lasers. It has been demonstrated that this technique enables multi-species measurements at rapid acquisition rates. The analysis of numerical simulations of the supersonic hydrogen combustion flow with respect to the absorption spectral distribution lead to the selection of frequency combs within the $1760 \pm 30 \mathrm{~cm}^{-1}$ wavenumber region which contains strong $\mathrm{NO}$ and $\mathrm{H}_{2} \mathrm{O}$ spectral lines. A method to obtain integrated line of sight spectral absorption spectra was created. This method allows to include the boundary layer effects in the analysis and it was demonstrated to be necessary for a more exact comparison. A calibration method was reported and its importance was shown with the help of parametric studies of the absorption line spectra.

\section{References}

1. Faber, J., et al.: Lower NOx at Higher Altitudes Policies to Reduce the Climate Impact of Aviation NOx Emission. Technical report, CE Delft (2008)

2. Kochanov, R.V., et al.: HITRAN application programming interface (HAPI): a comprehensive approach to working with spectroscopic data. J. Quant. Spectrosc. Radiat. Transfer 177, 15-30 (2016)

3. Fortier, T., Baumann, E.: 20 years of developments in optical frequency comb technology and applications. Commun. Phys. 2(153) (2019)

4. The Nobel Prize in Physics 2005. Nobel Media AB 2020. https://www.nobelprize. org/prizes/physics/2005/summary/. Accessed 19 Oct 2020

5. Faist, J., et al.: Quantum cascade laser frequency combs. Nanophotonics 5(2), 272-291 (2016) 
6. Probst, R.A.: Laser Frequency Combs for Astronomy. PhD thesis, Fakultät für Physik der Ludwig-Maximilians-Universität, München, 8 May 2015

7. Hugi, A., et al.: Mid-infrared spectrometer featuring $\mu$-second time resolution based on dual-comb quantum cascade laser frequency combs. In: 2017 Conference on Lasers and Electro-Optics (CLEO), pp. 14-19. IEEE, May 2017

8. Overton, G.: Spectrometers: Mid-IR spectrometer uses quantum-cascade laser frequency combs, 10 May 2017. https://www.laserfocusworld.com/testmeasurement/spectroscopy/article/16548279/spectrometers-midir-spectrometeruses-quantumcascade-laser-frequency-combs. Accessed 5 Oct 2020

9. IRsweep. IRis-F1 spectrometer ASC/PSC variants, 2 edition, September 2019. https://s3-eu-west-1.amazonaws.com/mediahomepage.irsweep.com/resources/ manuals/IRis-F1_UserManual_v2.pdf. Accessed 28 Aug 2020

10. Stalker, R.J.: A study of the free-piston shock tunnel. AIAA J. 5(12) (1967)

11. Hannemann, K., et al.: The high enthalpy shock tunnel Göttingen of the German aerospace center (DLR). J. Large-scale Res. Facil. 4(A133) (2018)

12. Hannemann, K., Martinez Schramm, J.: High enthalpy, high pressure short duration testing of hypersonic flows. Springer Handbook of Experimental Fluid Mechanics. Springer-Verlag, Berlin Heidelberg, January 2007

13. Steelant, J., Langener, T.: The LAPCAT-MR2 hypersonic cruiser concept. In: ICAS-2014-0428, 29th Congress of the International Council of the Aeronautical Sciences, St. Petersburg, pp. 7-12 September 2014

14. Roncioni, P., et al.: Numerical simulations and performance assessment of a scramjet powered cruise vehicle at mach 8. J. Aerospace Sci. Technol. 42, 218-228 (2015)

15. Langener, T., et al.: Layout and design verification of a small scale scramjet combustion chamber. In: Proceedings 21st International Symposium on Airbreathing Engines, Busan, Korea, 9-13 September 2013

16. Martinez Schramm, J., et al.: Ground testing synthesis of the LAPCAT II small scale flight experiment configuration scramjet flow path. In: 20th AIAA International Space Planes and Hypersonic Systems and Technologies Conferences, Glasgow, Scotland, 6-9 July 2015 